\title{
Adaptive Hybrid Position/Force Control for Robotic Manipulators with Compliant Links
}

\author{
Jung-Hua Yang ${ }^{1} \&$ Feng-Li Lian ${ }^{1} \&$ Li-Chen Fu ${ }^{1,2}$ \\ 1. Department of Electrical Engineering \\ 2. Department of Computer Science and Information Engineering \\ National Taiwan University, Taipei, Taiwan, R.O.C.
}

\begin{abstract}
In this paper, we tackle the problem of nonlinear adaptive hybrid control of constrained robots with flexible links. According to the physical properties of a flexible manipulator, a two time-scale approach, namely, singular perturbation approach, is further utilized for thorough analysis and general controller design. It is shown that asymptotic motional tracking can be effectively achieved, whereas the force regulation errors can be made arbitrarily small. For demonstration of the controller performance, experiments of a two-link flexible manipulator are performed for the proposed controller and satisfactory results are observed.
\end{abstract}

\section{INTRODUCTION}

Constrained robotic systems, by definition, require the manipulation tasks involve robot arms which are intersected with the environment. In the early development of constrained robotic systems, both robot and environment are assumed to be rigid. From theoretical point of view, this kind of problems can be relatively more tractable. However, it might be unrealistic, from application point of view, to apply such kind of machinery. If both are rigid as assumed, then one of them can very likely incur damage to the other due to the inevitable uncertainties. However, in many industrial applications such as grinding, deburring, etc., the environments are quite stiff in general, and hence it may be more feasible to adopt flexible manipulators in such a constrained environment. That therefore calls for development of a proper control for constrained robotic systems employing flexiblelink manipulator.

In the literatures, many fundamental issues on this regard have been extensively studied, such as impact analysis and contact force regulation, compliant force regulation, and force/position control during constrained motion. In [11], a standard approach for constrained manipulation have been developed, in which a systematic way is employed to reduce the system dynamics into lower-order ones and then a nonlinear feedback controller is designed to deal with the constrained system. Other controller designs based on the theory of variable structure systems [15], learning algorithm [5], and parallel approach [3] haev also been developed in the past. Jean and $\mathrm{Fu}$ [4] proposed an adaptive hybrid control scheme for the constrained robots based on both Lagrange and Newton-Euler dynamics formulations, and Stepanenko and Su [14] developed a controller which can adaptively tune the gains of the variable structure scheme.

For industrial applications, many extensive studies of flexible manipulators have been carried out. Dynamic models of multilink flexible manipulators are completely derived by Book [1] and Luca and Siciliano [9]. Many nonlinear control schemes such as those using computed torque, inverse dynamics, and feedback linearization, [8], [13], [16], have all been thoroughly developed for multilink flexible manipulators in the past decades. Extensive experimental studies of twolink flexible manipulators have been demonstrated in [2] and [12].

However, for the constrained flexible manipulators, there are relatively fewer results that have been presented. So far, Matsuno and Asano [10] and Lew and Book [7] have proposed hybrid controllers based on the quasi-static condition respectively dealing with single contact and multiple contacts. Matsuno and Yamamoto [10] dealt with the dynamic hybrid control on the basis of the singular perturbation method. The nonlinear inversion of the $1 / O$ map along with a linear feedback stabilization is studied in [17].

In this paper, a reduced model in Cartesian space is derived due to the constraints imposed on the end-effector. Based on singular perturbation theory, an adaptive controller is designed from the set of reduced dynamical equations. In the Advanced Control Laboratory (ACL) at National Taiwan University (NTU), a 2-link planar flexible robot has been built up to demonstrate the performance of the proposed controller, and experimental results have validated the effectiveness of the proposed controller.

This paper is organized as follow: Section 2 presents the dynamical model of a constrained flexible manipulator. In this section, we reformulate the dynamical model into Cartesian space and further reduce the original system into two subsystems, namely, fast subsystem and slow subsystem. In Section 3, an adaptive composite controller is developed based on the formulation of these the two subsystems. Experimental results are shown in Section 4 to demonstrate the controlled performance. Finally, some conclusions are given in Section 5. 


\section{Problem Formulation}

In this section, we consider the flexible manipulator whose end-effector is in contact with the environment modeled as a rigid frictionless surface. In the following derivation, we will use the subscripts $r$ and $f$ to denote the rigid-mode part and flexible-mode part, respectively. The dynamics of the constrained flexible manipulator can be derived by using the Lagrangian formulation via the assumed mode method which appear in the following form:

$$
\begin{array}{r}
{\left[\begin{array}{cc}
M_{r r} & M_{r f} \\
M_{f r} & M_{f f}
\end{array}\right]\left[\begin{array}{c}
\ddot{q}_{r} \\
\ddot{q}_{f}
\end{array}\right]+\left[\begin{array}{cc}
C_{r r} & C_{r f} \\
C_{f r} & C_{f f}
\end{array}\right]\left[\begin{array}{c}
\dot{q}_{r} \\
\dot{q}_{f}
\end{array}\right]} \\
+\left[\begin{array}{c}
G_{1} \\
G_{2}+K q_{f}
\end{array}\right]=\left[\begin{array}{c}
\tau+A_{1}^{T} \lambda \\
A_{2}^{T} \lambda
\end{array}\right]
\end{array}
$$

with

$$
\Phi\left(x\left(q_{r} q_{f}\right)\right)=\Phi^{\prime}\left(q_{r} q_{f}\right)=0
$$

where $A_{1}=\frac{\partial \Phi^{\prime}}{\partial q_{r}}, A_{2}=\frac{\partial \Phi^{\prime}}{\partial q_{f}}, x, q_{r} \in R^{n}, q_{f} \in R^{n_{f}}, \Phi: R^{n} \rightarrow$ $R^{m}$, and $\Phi^{\prime}: R^{N} \rightarrow R^{m}$ with $N=n+n_{f}$. For convenience, we first define the inverse of the inertia matrix $M$ as:

$$
\left[\begin{array}{ll}
M_{r r} & M_{r f} \\
M_{f r} & M_{f f}
\end{array}\right]^{-1}=\left[\begin{array}{ll}
H_{11} & H_{12} \\
H_{12} & H_{22}
\end{array}\right]
$$

so that (1) becomes

$$
\begin{aligned}
& \ddot{q}_{r}=C_{11} \dot{q}_{r}+C_{12} \dot{q}_{f}+g_{1}+H_{11} \tau+a_{1} \lambda \\
& \ddot{q}_{f}=C_{21} \dot{q}_{r}+C_{22} \dot{q}_{f}+g_{2}+H_{21} \tau+a_{2} \lambda
\end{aligned}
$$

where

$$
\begin{aligned}
{\left[\begin{array}{ll}
C_{11} & C_{12} \\
C_{21} & C_{22}
\end{array}\right] } & =-\left[\begin{array}{ll}
H_{11} & H_{12} \\
H_{21} & h_{22}
\end{array}\right]\left[\begin{array}{ll}
C_{r r} & C_{r f} \\
C_{f r} & C_{f f}
\end{array}\right] \\
{\left[\begin{array}{l}
g_{1} \\
g_{2}
\end{array}\right] } & =-\left[\begin{array}{ll}
H_{11} & H_{12} \\
H_{21} & h_{22}
\end{array}\right]\left[\begin{array}{l}
G_{1} \\
G_{2}
\end{array}\right] \\
{\left[\begin{array}{l}
a_{1} \\
a_{2}
\end{array}\right] } & =\left[\begin{array}{ll}
H_{11} & H_{12} \\
H_{21} & h_{22}
\end{array}\right]\left[\begin{array}{l}
A_{1}^{T} \\
A_{2}^{T}
\end{array}\right]
\end{aligned}
$$

As a matter of fact, when a manipulator is constrained by its environment, it is more convenient and realistic to use the coordinates in Cartesian space (i.e. task space) rather than the joint configuration space. Thus, in the following derivation, we will derive the equations of motion in Cartesian coordinates. Therefore, let the position of the end-effector be described as $x=X(q)$, where $X: R^{N} \rightarrow R^{n}, q^{T}=\left[\begin{array}{ll}q_{r}^{T}, & q_{f}^{T}\end{array}\right]^{T}$. If we take the first- and the second- time derivative of $x$, we will then have the relations of velocities and accelerations in joint space and in Cartesian space as follows:

$$
\begin{aligned}
& \dot{x}=J_{r} \dot{q}_{r}+J_{f} \dot{q}_{f} \\
& \ddot{x}=J_{r} \ddot{q}_{r}+J_{f} \ddot{q}_{f}+\dot{J}_{r} \dot{q}_{r}+\dot{J}_{f} \dot{q}_{f}
\end{aligned}
$$

where $J_{r}=\frac{\partial X}{\partial q_{r}}, J_{f}=\frac{\partial X}{\partial q_{f}}$. Without loss of generality, we have assumed that the flexible manipulator is non-redundant with respect to the rigid part, which implies $J_{\tau}$ is invertible for almost all $q \in R^{N}$, except maybe when $g_{r}$ is at certain configurations. For simplicity, in the rest of this paper, we will assume that the manipulator will be operated solely in the region where $J_{r}$ is uniformly invertible, which is doable if the desired motion trajectory can be somewhat carefully chosen. Therefore, we can obtain

$$
\begin{aligned}
\ddot{x}= & D_{1}\left(q_{r}, q_{f}, \dot{q}_{r}, \dot{q}_{f}\right) \dot{x}+D_{2}\left(q_{r}, q_{f}, \dot{q}_{r}, \dot{q}_{f}\right) \dot{q}_{f}+D_{3}\left(q_{r}, q_{f}\right) \\
& +D_{4}\left(q_{r}, q_{f}\right) K q_{f}+D_{5}\left(q_{r}, q_{f}\right) \tau+D_{6}\left(q_{r}, q_{f}\right) \lambda \\
\ddot{q}_{f}= & E_{1}\left(q_{r}, q_{f}, \dot{q}_{r}, \dot{q}_{f}\right) \dot{x}+E_{2}\left(q_{r}, q_{f}, \dot{q}_{r}, \dot{q}_{f}\right) \dot{q}_{f}+E_{3}\left(q_{r}, q_{f}\right) \\
& +E_{4}\left(q_{r}, q_{f}\right) K q_{f}+E_{5}\left(q_{r}, q_{f}\right) \tau+E_{6}\left(q_{r}, q_{f}\right) \lambda
\end{aligned}
$$

where

$$
\begin{aligned}
& D_{1}=\left(J_{r} C_{11}+J_{f} C_{21}+\dot{J}_{r}\right) J_{r}^{-1}, \\
& D_{2}=\left(J_{r} C_{12}+J_{f} C_{22}+\dot{J}_{f}\right)-\left(J_{r} C_{11}+J_{f} C_{21}+\dot{J}_{r}\right) J_{r}^{-1} J_{f}, \\
& D_{3}=-J_{r}\left(H_{11} G_{1}+H_{12} G_{2}\right)-J_{f}\left(H_{21} G_{1}+H_{22} G_{2}\right), \\
& D_{4}=-\left(J_{r} H_{12}+J_{f} H_{22}\right), \\
& D_{5}=\left(J_{r} H_{11}+J_{f} H_{21}\right), \\
& D_{6}=\left(J_{r} a_{1}+J_{f} a_{2}\right), \\
& E_{1}=C_{21} J_{r}^{-1} \\
& E_{\dot{2}}=\left(C_{22}-C_{21} J_{r}^{-1} J_{f}\right), \\
& E_{3}=-\left(H_{21} G_{1}+H_{22} G_{2}\right), \\
& E_{4}=-H_{22}, \\
& E_{5}=H_{21}, \\
& E_{6}=a_{2} .
\end{aligned}
$$

Now, we are ready to formulate the above dynamic model into a singular perturbation form via the definitions $z=K q_{f}$ and $\tilde{K}=K \epsilon^{2}$ where $\epsilon^{2}$ is a common factor extracted from each entry of the matrix $K$, assumed to be small enough. Further, we define the variables $z_{1}$ and $z_{2}$ as $z_{1}=z$ and $z_{2}=\epsilon \dot{z}$, and similarly $y_{1}$ and $y_{2}$ as $y_{1}=x$ and $y_{2}=\dot{x}$. Given these definitions, the state space of the slow subsystem and of the fast subsystem can thus be derived from (8) and (9), respectively, as follows:

$$
\begin{aligned}
\dot{y_{1}} & =y_{2} \\
\dot{y_{2}} & =D_{1} y_{2}+\epsilon D_{2} \tilde{K}^{-1} z_{2}+D_{3}+D_{4} z_{1}+D_{5} \tau+D_{6} \lambda \\
\epsilon \dot{z}_{1} & =z_{2} \\
\epsilon \dot{z}_{2} & =\tilde{K}\left(E_{1} y_{2}+\epsilon E_{2} \tilde{K}^{-1} z_{2}+E_{3}+E_{4} z_{1}+E_{5} \tau+E_{6} \lambda\right)
\end{aligned}
$$

which amounts to the singular perturbation model of the flexible manipulator system. One should note that the matrix $K$ plays the role of a constant stiffness matrix and, hence, the overall system becomes stiffer if $K$ is uniformly larger or, equivalently, $\epsilon$ is made smaller. According to the singular perturbation theory, the model obtained above will tend to a rigid model provided the system rigidity gradually diminishes. It can be shown that as $\epsilon \rightarrow 0,(10)$ becomes the model of a rigid manipulator, i.e., as $\epsilon \rightarrow 0$, one can obtain

$$
\begin{aligned}
& \bar{z}_{2}=0 \\
& \bar{z}_{1}=-\bar{E}_{4}^{-1}\left[\bar{E}_{1} \bar{y}_{2}+\bar{E}_{3}+\bar{E}_{5} \bar{\tau}+\bar{E}_{6} \bar{\lambda}\right],
\end{aligned}
$$

and, hence, the rigid manipulator model can be readily derived as :

$$
\dot{\bar{y}}_{1}=\bar{y}_{2}
$$




$$
\begin{aligned}
\dot{\bar{y}}_{2}= & {\left[\dot{\bar{J}}_{r}-\bar{J}_{r} \bar{M}_{r r}^{-1} \bar{C}_{r r}\right] \bar{J}_{r}^{-1} \bar{y}_{2}-\bar{J}_{r} \bar{M}_{r r}^{-1} \bar{G}_{1}+\bar{J}_{r} \bar{M}_{r r}^{-1} \bar{\tau} } \\
& +\bar{J}_{r} \bar{M}_{r r}^{-1} \bar{A}_{1}^{T} \bar{\lambda}
\end{aligned}
$$

where we have used the relation $M_{r r}=\left(H_{11}\right.$ $\left.\mathrm{H}_{12} \mathrm{H}_{22}^{-1} \mathrm{H}_{21}\right)^{-1}$, and all the variables with overbar are simply to denote those in the situation where $\epsilon=0$.

For deriving the fast subsystem, we let the fast time-scale be $\mu=\frac{t}{\epsilon}$ and redefine the fast variables $\eta_{1}=z_{1}-\bar{z}_{1}$ and $\eta_{2}=z_{2}$. Thus, the fast subsystem can be derived as :

$$
\frac{d}{d \mu}\left[\begin{array}{l}
\eta_{1} \\
\eta_{2}
\end{array}\right]=\left[\begin{array}{cc}
0 & I \\
-\tilde{K} \bar{H}_{22} & 0
\end{array}\right]\left[\begin{array}{l}
\eta_{1} \\
\eta_{2}
\end{array}\right]+\left[\begin{array}{c}
0 \\
\tilde{K} \tilde{H}_{21}
\end{array}\right](\tau-\bar{\tau})
$$

or equivalently,

$$
\frac{d \eta}{d \mu}=\bar{A} \eta+\bar{B} \tau_{f}
$$

Note that $\tau_{f}$ is the control input to the fast subsystem. As opposed to the objective of designing the slow mode control, the fast mode control $\tau_{f}$ is devised to make the set point $\eta=0$ uniformly exponentially stable. Hereafter, we will separately design the control inputs $\bar{\tau}$ and $\tau_{f}$ corresponding to the slow and the fast subsystems, respectively.

Next, due to the existing constraints, we will reduce the set of original equations of motion into a more realistic form. First, we divide the state $\bar{x}$, or equivalently $\bar{y}_{1}$, in the slow subsystem into two parts, namely, $\bar{x}_{1}$ and $\bar{x}_{2}$, where $\bar{x}_{1} \in R^{m}$ and $\bar{x}_{2} \in R^{n-m}$, and assume the constraint (2) can be reexpressed as:

$$
\tilde{\Phi}(\bar{x})=\bar{x}_{1}-\bar{\Omega}\left(\bar{x}_{2}\right)=0,
$$

where $\bar{\Omega}$ is a nonlinear map form $R^{n-m}$ to $R^{m}$. Furthermore, we can obtain the velocity and acceleration relations between $\bar{x}_{1}$ and $\bar{x}_{2}$ as:

$$
\begin{aligned}
& \bar{x}_{1}=\bar{\Omega}\left(\tilde{x}_{2}\right) \\
& \dot{\bar{x}}_{1}=\bar{F}_{2} \dot{\bar{x}}_{2} \\
& \ddot{\bar{x}}_{1}=\bar{F}_{2} \ddot{\bar{x}}_{2}+\dot{\bar{F}}_{2} \dot{\bar{x}}_{2},
\end{aligned}
$$

where $\bar{F}_{2}=\frac{\partial \Omega}{\partial \bar{x}_{2}}$ is assumed to be of full rank. Then, we rewrite the state-space equations (13) into the differential equations in terms of the Cartesian state, $\bar{x}$, by premultiplying the equations by $\bar{J}_{r}^{-T} \bar{M}_{r r} \bar{J}_{r}^{-1}$ and then using the following relation

$$
\bar{A}_{1}=\frac{\partial \bar{\Phi}^{\prime}}{\partial \bar{q}_{r}}=\frac{\partial \bar{\Phi}}{\partial \bar{x}} \frac{\partial \bar{x}}{\partial \bar{q}_{r}}=\left[\begin{array}{ll}
I & -\bar{F}_{2}
\end{array}\right] \bar{J}_{r}
$$

so that the resulting equations become:

$$
\begin{array}{r}
\bar{J}_{r}^{-T} \bar{M}_{r r} \bar{J}_{r}^{-1} \overline{\bar{x}}+\left[\bar{J}_{r}^{-T} \bar{C}_{r r} \bar{J}_{r}^{-1}-\bar{J}_{r}^{-T} \bar{M}_{r r} \bar{J}_{r}^{-1} \dot{\bar{J}}_{r} \bar{J}_{r}^{-1}\right] \dot{\bar{x}} \\
+\bar{J}_{r}^{-T} \bar{G}_{1}=\bar{J}_{r}^{-T} \bar{\tau}+\left[\begin{array}{c}
I \\
-\bar{F}_{2}^{T}
\end{array}\right] \bar{\lambda} .
\end{array}
$$

For convenience of analysis, we can further partition the matrices and rewrite the above equations into the following form:

$$
\begin{gathered}
{\left[\begin{array}{ll}
\bar{M}_{11} & \bar{M}_{12} \\
\bar{M}_{21} & \bar{M}_{22}
\end{array}\right]\left[\begin{array}{c}
\ddot{\bar{x}}_{1} \\
\overline{\bar{x}}_{2}
\end{array}\right]+\left[\begin{array}{ll}
\bar{B}_{11} & \bar{B}_{12} \\
\bar{B}_{21} & \bar{B}_{22}
\end{array}\right]\left[\begin{array}{l}
\dot{\bar{x}}_{1} \\
\overline{\bar{x}}_{2}
\end{array}\right]} \\
+\left[\begin{array}{c}
\bar{G}_{11} \\
\bar{G}_{12}
\end{array}\right]=\left[\begin{array}{l}
\bar{f}_{1} \\
\bar{f}_{2}
\end{array}\right]+\left[\begin{array}{c}
\bar{\lambda} \\
-\bar{F}_{2} \bar{\lambda}
\end{array}\right],
\end{gathered}
$$

Clearly, $\bar{J}_{r}^{-T} \bar{M}_{r r} \bar{J}_{r}^{-1}$ is symmetric and positive definite. Now, if (17) is used to replace $\bar{x}_{1}, \dot{\bar{x}}_{1}, \overline{\bar{x}}_{1}$ in (19), and then premultiply the upper part of (19) by $\bar{F}_{2}^{T}$ and add the results to its lower part, then the following equations are obtained.

$$
\begin{aligned}
\bar{M}_{1} \ddot{\bar{x}}_{2}+\bar{C}_{1} \dot{\bar{x}}_{2}+\bar{G}_{11} & =\bar{f}_{1}+\bar{\lambda}, \\
\bar{M}_{2} \ddot{\bar{x}}_{2}+\bar{C}_{2} \dot{\bar{x}}_{2}+\bar{F}_{2} \bar{G}_{11}+\bar{G}_{12} & =\bar{F}_{2}^{T} \bar{f}_{1}+\bar{f}_{2} .
\end{aligned}
$$

where

$$
\begin{aligned}
\bar{M}_{1}= & \left(\bar{M}_{11} \bar{F}_{2}+\bar{M}_{12}\right), \\
\bar{M}_{2}= & \bar{F}_{2}^{T}\left(\bar{M}_{11} \bar{F}_{2}+\bar{M}_{12}\right)+\left(\bar{M}_{21} \bar{F}_{2}+\bar{M}_{22}\right), \\
\bar{C}_{1}= & \bar{M}_{11} \overline{\bar{F}}_{2}+\bar{B}_{11} \bar{F}_{2}+\bar{B}_{12}, \\
\bar{C}_{2}= & \bar{F}_{2}^{T}\left(\bar{M}_{11} \overline{\bar{F}}_{2}+\bar{B}_{11} \bar{F}_{2}+\bar{B}_{12}\right) \\
& +\left(\bar{M}_{21} \overline{\bar{F}}_{2}+\bar{B}_{21} \bar{F}_{2}+\bar{B}_{22}\right) .
\end{aligned}
$$

Thus, we can refer to the equations (20) and (21) as force part and motion part, respectively. In the next section, we will design the controller based on the reduced slow-subsystem (20), (21) and fast-subsystem (15).

\section{Controller Design}

Our objective is to design a hybrid controller to achieve asymptotic tracking of both unconstrained coordinates $x_{2}$ and constrained forces $\lambda$, i.e., to yield

$$
x_{2}(t) \rightarrow x_{2 d}(t) \text { and } \lambda(t) \rightarrow \lambda_{d}(t) \text { as } t \rightarrow \infty .
$$

Before we proceed to present the controller design, we will summarize some useful dynamical properties of the flexible manipulators in the following proposition.

Proposition 3.1: For the constrained flexible manipulator described in the previous section, the following properties [4] will hold.

1) $\bar{M}_{2}$ is symmetric and positive definite.

2) By a proper choice of $C(q, \dot{q})$ to define $\bar{C}_{2}$, the matrix $\bar{M}_{2}-2 \bar{C}_{2}$ is skew-symmetric.

3) There exist some constant vectors $\theta_{1}^{*}$ and $\theta_{2}^{*}$ such that

$$
\begin{aligned}
& \bar{M}_{1} \dot{u}+\bar{C}_{1} u+\bar{G}_{11}=\bar{w}_{1}^{T} \theta_{1}^{*} \\
&\left(\bar{M}_{21} \bar{F}_{2}+\bar{M}_{22}\right) \dot{u}+\left(\bar{M}_{21} \dot{\bar{F}}_{2}+\bar{B}_{21} \bar{F}_{2}+\bar{B}_{22}\right) u \\
&+\bar{G}_{12}=\bar{w}_{2}^{T} \theta_{2}^{*},
\end{aligned}
$$

where $\bar{w}_{1}$ and $\bar{w}_{2}$ are known functions of their arguments.

Now, we are ready to introduce process of designing a controller for the manipulator system in the following.

\subsection{Slow Subsystem Controller}

We first define the auxiliary signal $\vec{s}$ as

$$
\bar{s}=\dot{\tilde{x}}_{2}+K_{r} \tilde{x}_{2},
$$

where $\tilde{x}_{2}=\bar{x}_{2}-x_{2 d}$ is the tracking error and $K_{r}$ is some positive constant. Let the control laws be designed as:

$$
\begin{aligned}
& \bar{f}_{1}=-\bar{w}_{1}^{T} \hat{\theta}_{1}+\kappa, \\
& \bar{f}_{2}=-\bar{w}_{2}^{T} \hat{\theta}_{2}-F_{2}^{T} \kappa-K_{p} \bar{s},
\end{aligned}
$$


for (20) and (21), respectively, where $\kappa=K_{f}\left(\hat{\lambda}-\lambda_{d}\right)-\hat{\lambda}$, $K_{f}>0, K_{P}>0$, and $\hat{\theta}_{i}, \hat{\lambda}$ denote the estimates of system parameters $\theta_{i}, i=1,2$, and the measurement of contact force $\lambda$. Let the parameter adaptation law be devised as:

$$
\left[\begin{array}{c}
\dot{\hat{\theta}}_{1} \\
\dot{\hat{\theta}}_{2}
\end{array}\right]=\dot{\hat{\theta}}=\Gamma^{-1}(\bar{w} \bar{s}-\sigma \hat{\theta}),
$$

where $\Gamma>0, \bar{w}^{T}=\left[\bar{F}_{2}^{T} \bar{w}_{1}^{T}, \bar{w}_{2}^{T}\right]$, and $\sigma$ is some positive constant.

Accordingly, the error dynamics can be obtained by the following derivation with the help of Proposition 3.1 and (20), $(21)$, i.e.,

$$
\begin{aligned}
\bar{M}_{2} \dot{\bar{s}}+\bar{C}_{2} \bar{s}= & \bar{M}_{2} \ddot{\bar{x}}_{2}+\bar{C}_{2} \dot{\bar{x}}_{2}+\bar{F}_{2}^{T} \bar{G}_{11}+\bar{G}_{12} \\
& +\bar{M}_{2}\left(-\ddot{x}_{2 d}+K_{r}\left(\overline{\bar{x}}_{2}-\dot{x}_{2 d}\right)\right) \\
& +\bar{C}_{2}\left(-\dot{x}_{2 d}+K_{r}\left(\bar{x}_{2}-x_{2 d}\right)\right)-\bar{F}_{2}^{T} \bar{G}_{11}-\bar{G}_{12} \\
= & \bar{F}_{2}^{T} \bar{f}_{1}+\bar{f}_{2}+\bar{F}_{2}^{T} \bar{w}_{1}^{T} \theta_{1}^{*}+\bar{w}_{2}^{T} \theta_{2}^{*} \\
= & {\left[\bar{F}_{2}^{T} \bar{w}_{1}^{T}, \bar{w}_{2}^{T}\right] \tilde{\theta}-K_{p} \bar{s} } \\
\bar{M}_{1} \dot{\bar{s}}+\bar{C}_{1} \bar{s}= & \bar{M}_{1} \ddot{\bar{x}}_{2}+\bar{C}_{1} \dot{\bar{x}}_{2}+\bar{G}_{11} \\
& +\bar{M}_{1}\left(-\ddot{x}_{2 d}+K_{r}\left(\dot{\bar{x}}_{2}-\dot{x}_{2 d}\right)\right) \\
& +\bar{C}_{1}\left(-\dot{x}_{2 d}+K_{r}\left(\bar{x}_{2}-x_{2 d}\right)\right)-\bar{G}_{11} \\
= & \bar{f}_{1}+\bar{w}_{1}^{T} \theta_{1}^{*}+\bar{\lambda} \\
= & \bar{w}_{1}^{T} \tilde{\theta}_{1}+K_{f}\left(\hat{\lambda}-\lambda_{d}\right)+(\bar{\lambda}-\hat{\lambda}) \\
= & \bar{w}_{1}^{T} \tilde{\theta}_{1}+K_{f}\left(\bar{\lambda}-\lambda_{d}\right)+\left(1-K_{f}\right)(\bar{\lambda}-\hat{\lambda}), \quad(29)
\end{aligned}
$$

where $\tilde{\theta}=\theta^{*}-\hat{\theta}$ is the parameter estimation error and $\tilde{\lambda}=$ $\bar{\lambda}-\lambda_{d}$ is the force tracking error. If we choose the Lyapunov function candidate $\bar{V}_{1}$ as:

$$
\bar{V}_{1}=\frac{1}{2} \bar{s}^{T} \bar{M}_{2} \bar{s}+\frac{1}{2} \tilde{\theta}^{T} \Gamma \tilde{\theta},
$$

and take its time derivative along the trajectories of (28) and (29), then, by virtue of Proposition 1, we have

$$
\begin{aligned}
\frac{d}{d t} \bar{V}_{1} & =\bar{s}^{T} \bar{M}_{2} \dot{\bar{s}}+\frac{1}{2} \bar{s}^{T} \dot{\bar{M}}_{2} \bar{s}+\dot{\tilde{\theta}}^{T} \Gamma \tilde{\theta} \\
& =\bar{s}^{T} \bar{M}_{2} \dot{\bar{s}}+\bar{s}^{T} \bar{C}_{2} s-\dot{\hat{\theta}}^{T} \Gamma \tilde{\theta} \\
& =\bar{s}^{T}\left(\bar{M}_{2} \dot{\bar{s}}+\bar{C}_{2} \bar{s}\right)-\dot{\hat{\theta}}^{T} \Gamma \tilde{\theta} \\
& =\bar{s}^{T}\left(\bar{w}^{T} \tilde{\theta}-K_{p} \bar{s}\right)-\dot{\hat{\theta}}^{T} \Gamma \tilde{\theta} \\
& =\left(\bar{s}^{T} \bar{w}^{T}-\dot{\hat{\theta}}^{T} \Gamma\right) \tilde{\theta}-\bar{s}^{T} K_{p} \bar{s} \\
& =-\bar{s}^{T} K_{p} \bar{s}-\tilde{\theta}^{T} \sigma \tilde{\theta}+\tilde{\theta} \sigma \theta^{*} \\
& =-\bar{s}^{T} K_{p} \tilde{s}-\frac{1}{2} \sigma\|\tilde{\theta}\|^{2}+\frac{1}{2} \sigma\left\|\theta^{*}\right\|^{2} \\
& \leq-\alpha_{1} \psi^{2}(\xi)+\alpha_{0}(\sigma, \theta)
\end{aligned}
$$

where $\xi=\left[s^{T}, \tilde{\theta}^{T}\right]^{T}, \alpha_{1}$ is some positive constant, $\alpha_{0}\left(\sigma, \theta^{*}\right)=$ $\frac{1}{2} \sigma\left\|\theta^{*}\right\|^{2}$, and $\psi(\xi)$ is a continuous function of $\xi$ which vanishes only at $\xi=0$. Therefore, we can guarantee that $\bar{s}$ and $\tilde{\theta}$ will converge to a residual set with size $O\left(\alpha_{0}\right)$, and so are $\tilde{x}_{2}, \dot{\tilde{x}}_{2}$. In the following, we will show that $\bar{\lambda}$ is also a bounded signal. Consider the closed-loop dynamical equation (28), where $\dot{\bar{s}}$ can be represented as

$$
\dot{\bar{s}}=\ddot{\tilde{x}}_{2}+K_{\mathrm{r}} \dot{\tilde{x}}_{2}=\bar{M}_{2}^{-1}\left(-\bar{C}_{2} s+\bar{w}^{T} \tilde{\theta}-K_{p} \bar{s}\right) .
$$

We then apply (32) to (29) to obtain

$$
\begin{aligned}
& \bar{M}_{1} \bar{M}_{2}^{-1}\left(-\bar{C}_{2} \bar{s}+\bar{w}^{T} \tilde{\theta}-K_{p} \bar{s}\right)+\bar{C}_{1} \bar{s} \\
& =\bar{w}_{1}^{T} \tilde{\theta}_{1}+K_{f} \tilde{\lambda}+\left(1-K_{f}\right)(\bar{\lambda}-\hat{\lambda}),
\end{aligned}
$$

Assume that $\|\bar{\lambda}-\hat{\lambda}\| \leq \beta$ for some constant $\beta$ which denotes the possible bound on the force measurements error, then from equation (29), (33) we can obtain

$$
\begin{aligned}
& \left\|\hat{\lambda}-\lambda_{d}\right\| \leq K_{f}^{-1}(\alpha+\beta) \\
& \left\|\bar{\lambda}-\lambda_{d}\right\| \leq K_{f}^{-1} \alpha+\beta
\end{aligned}
$$

with the constant $\alpha$ satisfying

$$
\alpha=\left\|\bar{M}_{1} \bar{M}_{2}^{-1}\left(-\bar{C}_{2} \bar{s}+\bar{w}^{T} \tilde{\theta}-K_{p} \bar{s}\right)+\bar{C}_{1} s-\bar{w}_{1}^{T} \tilde{\theta}_{1}\right\|
$$

Remark : In (35), we have theoretically derived the relations between the force tracking and the force measurement error. It is therefore obvious that the force tracking error can be made arbitrarily small by enlarging $K_{f}$ if precise force measurement can be obtained.

\subsection{Fast-Subsystem Controller}

We first rewrite (15) in the fast time scale $\mu$ as follows:

$$
\frac{d \eta}{d \mu}=\bar{A} \eta+\vec{B} \tau_{f} .
$$

Since our control objective is to regulate the fast state $\eta$, here we design a robust regulator. For the full-order system being dominated by the slow subsystem, a state regulator must be devised to force $\eta \rightarrow 0$ as fast as possible. Within the boundary layer, the system matrices $\bar{A}$ and $\bar{B}$ can be substituted by $\bar{A}_{0}+\Delta \bar{A}_{0}$ and $\bar{B}_{0}+\Delta \bar{B}_{0}$, respectively, where $\bar{A}_{0}$ and $\bar{B}_{0}$ are nominal matrices with known elements plus the known bounds on $\left\|\Delta \bar{A}_{0}\right\|$ and $\left\|\Delta \bar{B}_{0}\right\|$. And, we further design a dynamic feedback controller as follows:

$$
\frac{d \tau_{f}}{d \mu}=\bar{F} \eta+\vec{G} \tau_{f}
$$

where the matrices $\vec{F}$ and $\vec{G}$ will be determined later, whereby the closed-loop system consisting of (36) and (37) becomes

$$
\frac{d \zeta}{d \mu}=\mathcal{A} \zeta+\mathcal{B} \zeta
$$

where $\zeta=\left[\eta^{T}, \tau_{f}^{T}\right]^{T}, \mathcal{A}=\left[\begin{array}{cc}\bar{A}_{0} & \bar{B}_{0} \\ \bar{F} & \bar{G}\end{array}\right]$, and $\mathcal{B}=$ $\left[\begin{array}{cc}\Delta \bar{A}_{0} & \Delta \bar{B}_{0} \\ 0 & 0\end{array}\right]$. The following theorem provides a condition under which the above design of the fast-subsystem controller may give the desirable result.

Theorem 3.2: If $\bar{F}$ and $\bar{G}$ are chosen such that $\mathcal{A}$ is Hurwitz and if there exist matrices $P, Q>0$ satisfying

$$
\mathcal{A}^{T} P+P \mathcal{A}=-Q
$$

and $\lambda_{\min }(Q)>2 \alpha\|P\|$, where $\|\mathcal{B}\| \leq \alpha$, then it is guaranteed that $\|\zeta\| \rightarrow 0$ exponentially. 
Proof: Let the Lyapunov function candidate $V_{2}$ be

$$
V_{2}=\frac{1}{2} \zeta^{T} P \zeta
$$

and take the fast time derivative of $V_{2}$ as follows:

$$
\begin{aligned}
\frac{d V_{2}}{d \mu} & =\frac{1}{2} \zeta^{T}\left(P \mathcal{A}+\mathcal{A}^{T} P\right) \zeta+\frac{1}{2} \zeta^{T}\left(P \mathcal{B}+\mathcal{B}^{T} P\right) \zeta \\
& \leq-\frac{1}{2} \lambda_{\min }(Q)\|\zeta\|^{2}+\|\alpha\|\|P\|\|\zeta\|^{2} \\
& =-\frac{1}{2}\left(\lambda_{\min }(Q)-2\|\alpha\|\|P\|\right)\|\zeta\|^{2} \\
& \leq-\epsilon \alpha_{2} \phi^{2}(\zeta)
\end{aligned}
$$

where $\alpha_{2}$ is some positive constant and $\phi(\zeta)$ is a continuous function which vanishes only at $\zeta=0$. Thus, through a Lyapunov theorem, this theorem can thus be concluded. This property will be useful in our later derivation of a composite controller.

\subsection{Composite Controller}

Consider the original system (10)-(11) with the composite control law $\tau=\bar{\tau}+\tau_{f}$. It can be shown that with such composite control law the original system will be uniformly ultimately bounded. The results are detailedly summarized in the following theorem.

Theorem 3.3 : Consider the system (10)-(11) with the composite control law $\tau=\bar{\tau}+\tau_{f}$ and the adaptation law (27). Then, all signals inside the system remain bounded and both position tracking errors and link vibration will converge into a residual set of a size which is an order of $\alpha_{0}$ and $\epsilon$, provided $\epsilon$ is sufficiently small.

Proof: The stability proof is omitted here due to limited space. One can see [18] for details.

Q.E.D.

\section{Experimental Verification}

\subsection{Apparatus}

A 2-link flexible manipulator has been set up and experimented in the Department of Electrical Engineering at $\mathrm{Na}$ tional Taiwan University (NTU). The 2-link flexible manipulator is a planar-type manipulator with two revolute joints which are perpendicular to the motional plane, see Fig.(1). The first link is driven by a D.C. motor with ratio $128: 1$, and the second link is driven by a D.C. Brushless motor with gear ratio 100:1. In adidion, the hub of the second joint of the manipulator is air-jetted in order to counteract the gravity. A PC 486-33 is used as the processor to implement the computation of the contrcl law and the adaptation law, in which the sampling rate is set to be $400 \mathrm{~Hz}$. The mathematical model of this flexible manipulator is derived based on the so-called assumed mode method, and the vibration modes (two for each link) are measured using strain gauges through some properly designed low-pass filter to account for the observation spillover.

\subsection{Experimental Results}

The constrained surface, which is made of rigid material, at the location $x=0.7$. The desired motion trajectory is taken to be the form of a fifth-order polynomial trajectory $y_{d}(t)=$ $0.5 r(t)$, where

$\begin{cases}r(t)=6\left(\frac{t}{T_{m}}\right)^{5}-15\left(\frac{t}{T_{m}}\right)^{4}+10\left(\frac{t}{T_{m}}\right)^{3} & \text { for } 0 \leq t \leq T_{m} \\ r(t)=1 & \text { for } T_{m} \leq t\end{cases}$

and $T_{m}=3 s e c$ is the expected duration of the motion. The desired contact force is $1 \mathrm{~N}$. One should note that, in this case $(x=0.7)$, the contact force $f$ is equal to the Lagrangian multiplier $\lambda$ since $\frac{\theta \Phi(x)}{\theta x}^{T} \lambda=f$ and $\Phi(x)=x-0.7$. The gain $K_{r}, K_{p}$, and $K_{f}$ are 4, 10, and 20, respectively. The results are plotted in Fig.(2)-Fig.(5), in which Fig.(2) is the tracking error of motion in y-direction, and Fig.(3) plots the force tracking error in $\mathrm{x}$-direction. The deflections are demonstrated in Fig.(4)-Fig.(5).

\section{Conclusions}

In this paper, an adaptive hybrid control law is task space is developed via singular perturbation approach. Under this formulation, the original full-order system is decomposed into a two time-scale system, namely, the slow subsystem and the fast subsystem. The experiments of a two-link flexible manipulator has been set up to demonstrate the satisfactory performance of the proposed controller.

\section{References}

[1] Book, W. J., "Recursive Lagrangian dynamics of flexible manipulator arms," Int. J. of Robot. Res., vol. 3, no. 3, Fall, pp. 87-101, 1984.

[2] Carusone, J., K. S. Buchan, and G. M. T. D'Eleuterio, "Experiments in end-effector tracking control for structurally flexible space manipulators," IEEE Trans. Robot. Automat., vol. 9, no. 5, pp. 553-560, 1993.

[3] Chiaverini, S. and L. Sciavicco, "The parallel approach to force/position control of robotic manipulators," IEEE Trans. Robot. Automat., vol. 9, no. 4, pp. 361-373, Aug. 1993.

[4] Jean, J.-H. and L.-C. Fu, "Adaptive hybrid control strategies for constrained robots," IEEE Trans. Robot. Automat., vol. 38, no. 4, pp. 598-603, April 1993.

[5] Jeon, D. and M. Tomizuka, "Learning hybrid force and position control of robot manipulators," IEEE Trans. Robot. Automat., vol. 9, no. 4, pp. 423-431, Aug. 1993.

[6] Kokotovic, P. V., A. Bensoussan, and G. Blankenship, "Singular perturbations and asymptotic analysis in control systems," Springer-Verlag, vol. 90, 1987.

[7] Lew, J. Y. and W. J. Book, "Hybrid control of flexible manipulators with multiple contact," Proc. IEEE Conf. Robot. Automat., pp. 242-247, 1993. 
[8] Lewis, F. L. and M. Vandegrift, "Flexible robot arm control by a feedback linearization/singular perturbation approach," Proc. IEEE Conf. Robot. Automat., pp. 729$736,1993$.

[9] Luca, D. A. and B. Siciliano, "Closed-form dynamic model of planar multilink lightweight robots," IEEE Trans. Syst. Man Cybernetics, vol. 21, no. 4, pp. 826839, July/August 1991.

[10] Matsuno, F. and K. Yamamoto, "Dynamic hybrid position/force control of a fiexible manipulator," Proc. IEEE Conf. Robot. Automat., pp. 462-467, 1993.

[11] McClamroch, N. H. and D. Wang, "Feedback stabilization and tracking of constrained robots," IEEE Trans. Automat. Contr., vol. 33, no. 5, pp. 419-426, May 1988.

[12] Paden, B., D. Chen, R. Ledesma, and E. Bayo, "Exponentially stable tracking control for multijoint flexiblelink manipulators," ASME J. Dyn. Syst. Meas. Contr., vol. 115, pp. 53-59, Mar. 1993.

[13] Pham, C. M., W. Khalil, and C. Chevallereau, "A nonlinear model-based control of flexible robots," Robotica, vol. 11, pp. 73-82, A pril, 1993.

[14] Stepanenko, Y. and C.-Y. Su, "An efficient adaptive variable structure tracking control strategy for constrained robots," Proc. 12th Int'l Federation of Automat. Contr., vol. 1, pp. 525-528, 1993.

[15] Su, C.-Y., T.-P. Leung, and Q.-J. Zhou, "Force/motion control of constrained robots using sliding mode," IEEE Trans. Automat. Contr., vol. 37, no. 5, pp. 668-672, May 1992.

[16] Yang, J.-H., F.-C. Liu, and L.-C. Fu, "Nonlinear control for flexible manipulators," Proc. IEEE Conf. Robot. Automat., 1994.

[17] Yim, W. and S. N. Singh, "Inverse forced-point control, zero dynamics and stabilization of constrained elastic robots," Proc. IEEE American Contr. Conf., pp. 2873$2877,1993$.

[18] Yang, J.-H, F.-L. Lian, and L.-C. Fu, "Nonlinear Adaptive Control for Constrained Flexible Manipulators", Technical Report, Electrical Engineering, National Taiwan University, Taiwan, R.O.C., 1994.

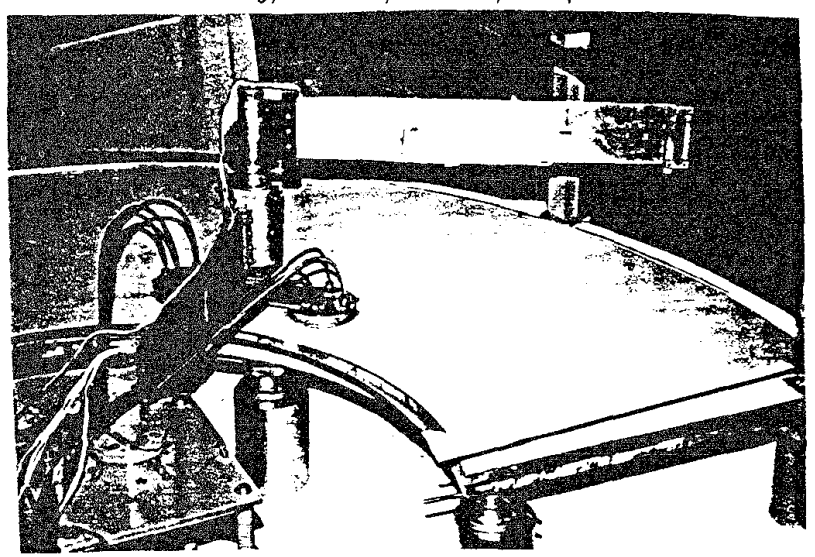

Fig.(1) Experimental Setup of the 2-link Flexible Arm

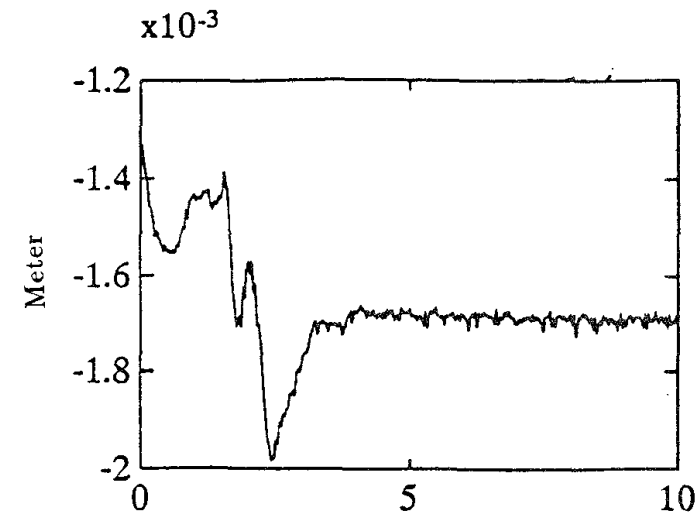

Fig.(2) Tracking Error of Tip Posïtion(Sec)

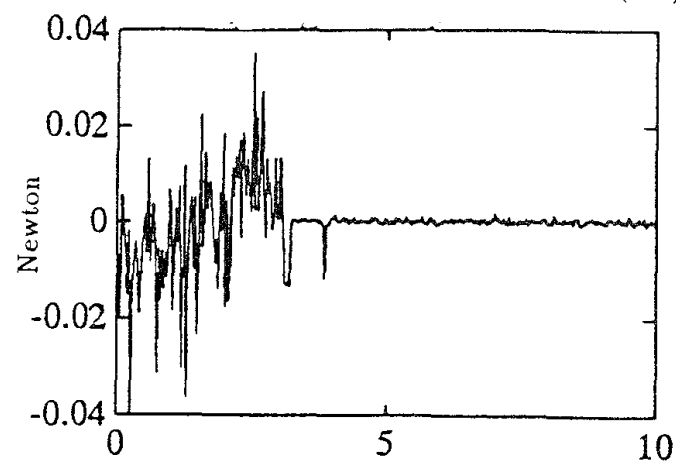

Fig.(3) Tracking Error of Contact Force(Sec)

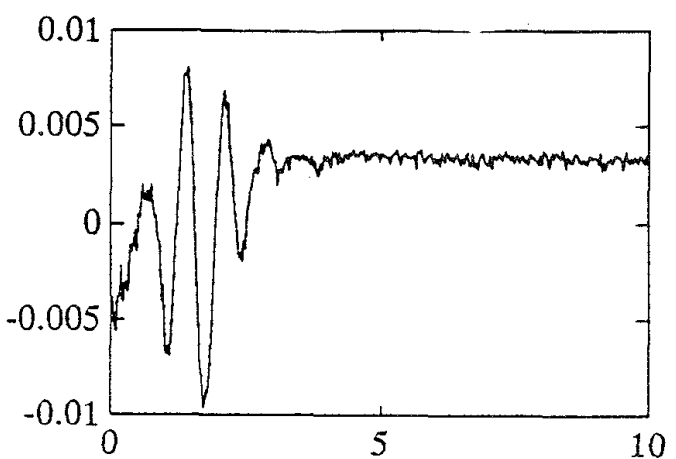

Fig. (4) 1st Vibration Mode of Link 1(Sec)

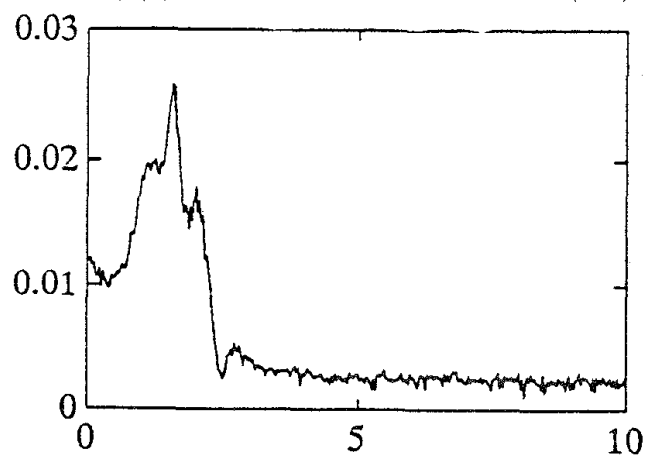

Fig.(5) 1st Vibration Mode of Link 2 (Sec) 